\title{
Comprar compulsivo: revisão sistemática das opções terapêuticas*
}

\section{Compulsive buying: systematic review of the therapeutic options}

\section{Helio Tonelli'; Cristiano Estevez Alvarez"'; Cristina Bertoluccill'; Dayane Diomario Rosa'V}

IPsiquiatra. Mestre em Farmacologia pela Universidade Federal do Paraná (UFPR), Curitiba, PR. Preceptor, responsável pela Disciplina de Farmacologia e pelo Ambulatório de Esquizofrenia Refratária da Residência em Psiquiatria do Hospital Nossa Senhora da Luz, Curitiba, PR.

IIPsiquiatra. Mestrando em Psiquiatria pela Universidade Federal de São Paulo (UNIFESP), São Paulo, SP. Coordenador da Disciplina de Medicina Baseada em Evidências da Residência em Psiquiatria do Hospital Nossa Senhora da Luz.

IIIPsiquiatra do Hospital Nossa Senhora da Luz.

IV Médica. Especializanda em Psiquiatria, Hospital Nossa Senhora da Luz.

Endereço para correspondência

Rev Psiquiatr RS. 2008;30(1 Supl)

\section{RESUMO}

O presente estudo inclui ensaios clínicos randomizados, controlados ou não, a respeito do tratamento farmacológico de comprar compulsivo, publicados em inglês ou em português nas bases MEDLINE, PsycINFO, Biological Abstracts e Cochrane Central Register of Controlled Trials. De todos os seis artigos selecionados, apenas dois eram estudos duplo-cegos controlados com placebo desde o princípio, e um terceiro era composto de uma fase inicial aberta seguida de uma segunda fase randomizada e placebo-controlada. É importante destacar que, em todos os estudos, pacientes com transtorno afetivo bipolar foram excluídos. Todos os ensaios envolveram amostras pequenas (variaram de 10 a 42 pacientes). De maneira geral, as evidências atualmente disponíveis a respeito do tratamento farmacológico de comprar compulsivo são pobres, em virtude da escassez de mais estudos metodologicamente bem conduzidos.

Descritores: Transtorno obsessivo-compulsivo, comportamento compulsivo, transtornos do controle de impulsos.

\section{ABSTRACT}

This study included randomized and non-randomized controlled trials on pharmacological treatment of compulsive buying published in English or Portuguese. Databases evaluated were MEDLINE, PsycINFO, Biological Abstracts and Cochrane Central Register of Controlled Trials. Of all six selected articles, only two were placebo-controlled randomized trials, and one had a first open-label phase followed by a second placebo-controlled phase. It is important to stress that all studies excluded bipolar patients. All the studies reviewed included small samples (from 10 to 42 patients). Current available evidence regarding pharmacological treatment of compulsive buying is poor due to the lack of studies using more rigorous methodology.

Keywords: Obsessive-compulsive disorders, compulsive behavior, impulse control disorders.

\section{NTRODUÇÃO}

Comprar compulsivo (CC), ou oniomania, é um transtorno classificado no Manual de Diagnóstico e Estatística das Perturbações Mentais (DSM-IV) ${ }^{1}$ na categoria "Transtornos do controle de impulsos não especificados", sendo caracterizado pela incapacidade de resistir a um impulso, tendência ou tentação para realizar um ato potencialmente nocivo ao indivíduo ou a terceiros.

Trata-se de um transtorno freqüentemente associado a complicações psicológicas, interpessoais, financeiras e legais ${ }^{2,3}$, cuja prevalência oscila entre 1,8 e $16 \%$ da população norte-americana adulta ${ }^{4}$.

A despeito de diversas modalidades de psicoterapia terem sido descritas como alternativas terapêuticas para CC, antidepressivos inibidores da recaptação de serotonina podem ser utilizados no tratamento do $\mathrm{CC}^{5}$.

O objetivo deste estudo é realizar uma revisão sistemática da literatura acerca do tratamento farmacológico do CC.

\section{MÉTODO}

Foram incluídos estudos clínicos randomizados, controlados ou não, a respeito do tratamento farmacológico do CC, publicados em inglês ou em português. Procuramos por estudos que avaliassem o tratamento psicofarmacológico de portadores de CC, realizando busca eletrônica por artigos disponíveis até junho de 2006, através de uma estratégia discriminada a seguir. 
MEDLINE: Foi utilizada a frase de busca: compulsive behavior/drug therapy (MeSH) OR compulsive behavior/prevention and control (MeSH) OR compulsive behavior/therapy (MeSH) OR impulse control disorders/drug therapy (MeSH) OR impulse control disorders/prevention and control (MeSH) OR impulse control disorders/therapy (MeSH) OR compulsive buying OR compulsive shopping OR oniomania OR pathological buying.

Para as bases Cochrane Central Register of Controlled Trials, PsycINFO, Biological Abstracts e LILACS, foi utilizada a seguinte frase: compulsive buying OR compulsive shopping OR oniomania OR pathological buying.

Obtidos os resumos, os artigos identificados foram avaliados independentemente pelos três autores para confirmar se realmente preenchiam os critérios de elegibilidade. Caso houvesse dúvida ou discordância entre os autores, o artigo era incluído. Em seguida, os artigos selecionados foram lidos, e aqueles que ainda não preenchessem os critérios foram excluídos.

Medidas de desfecho

As medidas de eficácia primária utilizadas nos estudos deveriam incluir a resposta clínica baseada em uma redução sintomática, como uma pontuação da Clinical Global Impression - Improvement Scale (CGI-I) de 2 (melhorado) ou 1 (muito melhorado).

\section{RESULTADOS}

A busca na base MEDLINE resultou em 1.122 artigos, dos quais foram selecionados seis ${ }^{6-11}$. A busca no PsycINFO resultou em 133 artigos, dos quais cinco foram incluídos ${ }^{6-9,11}$. Todos estes já tinham sido incluídos na busca pelo MEDLINE. A base Biological Abstracts retornou 21 artigos, dos quais foi selecionado $\mathrm{um}^{8}$, que também havia sido selecionado pelo MEDLINE e pelo PsycINFO. A busca via Cochrane Central Register of Controlled Trials forneceu quatro artigos ${ }^{7-9,11}$, todos também previamente selecionados via MEDLINE.

Dos artigos selecionados, apenas dois eram estudos duplo-cegos controlados com placebo desde o princípio 7,8 , ao passo que o estudo de Aboujaoude et al. ${ }^{10}$ é composto de uma fase inicial aberta, seguida de uma segunda fase randomizada e placebo-controlada. É importante destacar que, em todos os estudos, pacientes com transtorno afetivo bipolar foram excluídos através da utilização das entrevistas estruturadas Structured Clinical Interview for DSM-III-R/DSM-IV (SCID) ${ }^{6-8}$ e Mini-International Neuropsychiatric Interview, version 4.4 (MINI) ${ }^{9-11}$. Todos os ensaios envolveram amostras pequenas (variaram de 10 a 42 pacientes).

Black et al. ${ }^{7}$ realizaram um estudo aberto de 10 semanas usando fluvoxamina em doses flexíveis (até $300 \mathrm{mg} / \mathrm{dia}$ ). Na primeira semana do estudo, todos os pacientes receberam placebo. Nove dos 10 pacientes do estudo foram classificados como respondedores - melhora ? a 50\% na Yale-Brown Obsessive Compulsive Scale - Shopping Version (YBOCS-SV). O estudo apresenta limitações relativas ao seu desenho aberto.

Ninan et al. ${ }^{8}$ realizaram um estudo de 13 semanas com fluvoxamina (até $300 \mathrm{mg} /$ dia), duplo-cego, randomizado e controlado com placebo. Este ensaio envolveu 42 pacientes, dos quais 37 foram incluídos na análise de intenção de tratar. Não foram encontradas diferenças estatisticamente significativas entre fluvoxamina e placebo. Nesse estudo, os pacientes deveriam preencher um diário de compras. Os autores sugerem que a elevada resposta ao placebo poderia ser atribuída à utilidade do diário como uma intervenção comportamental.

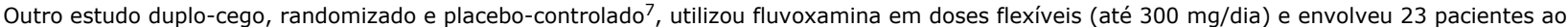
longo de 10 semanas. Os dois grupos mostraram melhora, entretanto, na maioria das medidas não houve diferença estatística. Os autores acreditam que o suporte psicoeducativo e a atenção dispensada aos pacientes podem ter contribuído para a alta taxa de resposta ao placebo.

O estudo aberto de Koran et al. ${ }^{9}$, de 12 semanas, incluiu 24 pacientes que utilizaram citalopram ( 20 a 60 mg/dia). Houve redução estatisticamente significativa nos escores de todas as medidas de eficácia, com uma taxa de resposta (CGI de 1 ou 2 ) de $71 \%$. Atenção clínica e registros de compras podem ter influenciado os resultados também neste estudo. Em seguida, foi realizado um estudo subseqüente ${ }^{10}$, aberto, que envolveu 23 pacientes em um seguimento de 1 ano. A maioria dos pacientes responsivos no final da fase aberta manteve a resposta, enquanto a maioria dos pacientes nãoresponsivos continuou a preencher os critérios diagnósticos para CC. Todavia, não houve associação, nos respondedores, com a manutenção ou não do citalopram.

Koran et al. ${ }^{11}$ realizaram um estudo em duas fases: uma fase aberta inicial de 7 semanas, com 24 pacientes usando citalopram (20 a 60 mg/dia), na qual houve uma taxa de resposta (CGI 1 ou 2 ou redução $\geq 50 \%$ na YBOCS-SV) de $63 \%$. No final da primeira fase, os 15 respondedores foram randomizados para receber citalopram ou placebo na segunda fase duplo-cega, com duração de 9 semanas. Os resultados da segunda fase mostraram uma taxa de recaída estatisticamente maior no grupo placebo do que no grupo citalopram (63 versus $0 \%$, respectivamente), além de diferença estatisticamente significativa nos escores YBOCS-SV entre os dois grupos.

\section{SCUSSÃO}

É possível afirmar, a partir dos resultados encontrados, que há poucos estudos duplo-cegos, randomizados e placebo-controlados a respeito do tratamento farmacológico do CC. Além disso, os três estudos que utilizaram essa metodologia $7,8,10$ têm um pequeno número de participantes ( 23,42 e 15 pacientes, respectivamente). Todavia, algumas considerações podem ser feitas levando-se em conta os resultados desses trabalhos.

Em primeiro lugar, todos os estudos selecionados envolveram antidepressivos inibidores seletivos da recaptação de serotonina, o que provavelmente reflete o conhecimento disponível a respeito das relações entre a circuitaria serotonérgica e comportamentos agressivos e impulsivos, bem como o efeito dessas drogas sobre esses comportamentos. Além disso, o CC tem sido relacionado a condições do espectro obsessivo-compulsivo, cuja resposta terapêutica aos inibidores seletivos de recaptação de serotonina está bem estabelecida. No entanto, os sistemas cerebrais subjacentes ao transtorno obsessivo-compulsivo (TOC) são muito complexos e não envolvem apenas a neurotransmissão serotonérgica, de forma que mais pesquisas envolvendo tanto a relação do CC com o TOC e condições correlatas quanto alterações de outros sistemas de neurotransmissão no CC devem ser feitas, a fim de se entender melhor o CC.

Em segundo lugar, outra hipótese considera que as dependências comportamentais (categoria à qual pertence o CC) e os transtornos relacionados ao uso de substâncias compartilhem tanto comemorativos clínicos (como tolerância e abstinência) quanto circuitos cerebrais ${ }^{12}$. Esses circuitos relacionamse principalmente aos sistemas neurais de recompensa, cujo principal neurotransmissor envolvido é a dopamina. Postulou-se que os dois grupos de dependências (comportamental e química) poderiam envolver alterações de grupos de genes relacionados a vários neurotransmissores envolvidos direta ou indiretamente com a regulação da dopamina, produzindo uma síndrome de deficiência de recompensa ${ }^{13}$. No entanto, vários neurotransmissores estão por trás da regulação da dopamina nos mecanismos fisiológicos de recompensa além da serotonina - noradrenalina, opióides, ácido gamaaminobutírico e canabinóides endógenos ${ }^{13}$-, tornando ainda mais complexa a compreensão desses sistemas e as conseqüências comportamentais de sua desregulação. Para tornar a tarefa de quem tente entender as relações das dependências comportamentais com os transtornos relacionados ao uso de substâncias ainda mais árida, tanto uma quanto outra estão associadas a alto grau de comorbidade psiquiátrica. 
Em terceiro lugar, parece ser grande a interferência de medidas não-farmacológicas na melhora de alguns pacientes dos estudos avaliados. Conforme sugerem Black et al. ${ }^{7}$, Ninan et al. ${ }^{8}$ e Koran et al. ${ }^{9}$, a automonitorização através de diários e o ambiente de maior atenção proporcionado aos indivíduos pesquisados, seja em virtude das entrevistas realizadas periodicamente, seja pelo próprio enfoque psicoeducativo que receberam ao participarem dos estudos, podem ter sido fatores importantes de melhora nesses casos, em detrimento do uso de um psicofármaco. De fato, da mesma forma que os transtornos por uso de substâncias, os circuitos cerebrais envolvidos nos sintomas do CC talvez sejam responsivos também a intervenções nãofarmacológicas.

Enfim, resta lembrar que a nosografia do CC ainda é controversa: seria mesmo uma categoria diagnóstica válida? Em caso afirmativo, existe realmente semelhança neurobiológica com algum grupo de transtornos, como os transtornos do espectro obsessivo-compulsivo ou os transtornos relacionados ao uso de substâncias?

Em suma, as evidências disponíveis a respeito do tratamento farmacológico do CC são, ainda, pobres, em virtude da escassez de estudos metodologicamente bem conduzidos. Além disso, até agora os estudos enfocaram apenas drogas com efeito serotonérgico, e alguns deles envolveram procedimentos de caráter psicoeducativo, que por si só podem ter tido um efeito terapêutico sobre o CC.

A Tabela 1 em anexo resume as principais características dos estudos selecionados.

\begin{tabular}{|c|c|c|c|c|c|c|c|}
\hline Estudo & Desenho & $\mathrm{n}$ & Intervenção & Dose & Duraçäo & Medidas de avaliaçâo & Resultado \\
\hline Black et al. ${ }^{6}$ & Aberto & 10 & Fluvoxamina & Até $300 \mathrm{mg} / \mathrm{dia}$ & 10 semanas & $\begin{array}{l}\text { YBOCS-SV, NIMHOCS, CGI, } \\
\text { Patient Self-Rating Scale, } \\
\text { Sheehan Disability Scale }\end{array}$ & $\begin{array}{l}\text { Grupo fluvoxamina apresentou mel hora } \\
\text { estatisticamente significativa e em quase todas } \\
\text { as medidas de avaliacão }\end{array}$ \\
\hline Ninan et al. ${ }^{8}$ & $\begin{array}{l}\text { Duplo-cego, randomizado e } \\
\text { controlado com placebo }\end{array}$ & 42 & Fluvoxamina & Até $300 \mathrm{mg} / \mathrm{dia}$ & 13 semanas & $\begin{array}{l}\text { YBOCS-SV, CGI, GAF, HAM-D, } \\
\text { diário de compras }\end{array}$ & $\begin{array}{l}\text { Mehora nos grupos placebo e fluvoxamina, } \\
\text { sem diferenças estatisticamente significativas } \\
\text { entre eles }\end{array}$ \\
\hline Black et al. ${ }^{7}$ & $\begin{array}{l}\text { Duplo-cego, randomizado e } \\
\text { controlado com placebo }\end{array}$ & 23 & Fluvoxamina & Até $300 \mathrm{mg} / \mathrm{dia}$ & 10 semanas & $\begin{array}{l}\text { Primárias: YBOCS-SV, NIMHOCS, } \\
\text { CGI }\end{array}$ & $\begin{array}{l}\text { Mehora os grupos placebo e fluvoxamina, } \\
\text { sem diferenças estatisticamente significativas } \\
\text { na grande maioria das medidas }\end{array}$ \\
\hline Koran et al. 9 & Aberto & 24 & Citalopram & 20 a $60 \mathrm{mg} / \mathrm{dia}$ & 12 semanas & Primárias: YBOCS-SV, CGI-I & $\begin{array}{l}\text { Melhora estatisticamente significativa do grupo } \\
\text { cital lopram em todas as modidas de avaliacäo }\end{array}$ \\
\hline Aboujaoude et al. ${ }^{10}$ & $\begin{array}{l}\text { Estudo naturalistico realizado após } \\
\text { um estudo aberto de } 3 \text { meses, que } \\
\text { dividiu os pacientes em } \\
\text { responsivos e nâo-responsivos }\end{array}$ & 23 & Citalopram & 20 a 60 mg/dia & 1 ano & $\begin{array}{l}\text { Critérios de McElroy, YBOCS-SV, } \\
\text { MADRS }\end{array}$ & $\begin{array}{l}\text { A maioria dos pacientes responsivos no final } \\
\text { da fase aberta manteve a resposta em cada } \\
\text { follow-un-up, independentemente da continuacãa } \\
\text { ou näo do citalopram, enquanto a maioria dos } \\
\text { pacientes não-responsivos continuou a } \\
\text { preencher os criterios diagnósticos para CC }\end{array}$ \\
\hline \multirow[t]{2}{*}{ Koran et al. ${ }^{11}$} & Fase 1: aberto & 24 & Citalopram & 20 a 60 mg/dia & 7 semanas & YBOCS-SV, CGI & $\begin{array}{l}\text { Taxa de resposta de } 63 \% \text {, com melhora } \\
\text { estatisticamente significativa do grupo } \\
\text { citalopram }\end{array}$ \\
\hline & $\begin{array}{l}\text { Fase 2: duplo-cego, randomizado e } \\
\text { controlado com placebo }\end{array}$ & 15 & Citalopram & $\begin{array}{l}\text { Mesma usada na } \\
\text { semana } 7\end{array}$ & 9 semanas & $\begin{array}{l}\text { Primárias: taxa de recaída, } \\
\text { YBOCS-SV }\end{array}$ & $\begin{array}{l}\text { Taxa de recaída estatisticamente maior no } \\
\text { grupo placebo do que no grupo cital opram }\end{array}$ \\
\hline
\end{tabular}

\section{REFERÊNCI AS}

1. American Psychiatric Assciation. DSM - IV - Manual diagnóstico e estatísticos de transtornos mentais. $4^{a}$ ed. Porto Alegre. Artmed; 1995.

2. McElroy SL, Keck PE Jr., Pope HG Jr., Smith JM, Strakowski SM. Compulsive buying: a report of 20 cases. J Clin Psychiatry. 1994;55(6):242-8.

3. Schlosser S, Black DW, Repertinger S, Freet D. Compulsive buying. Demography, phenomenology, and comorbidity in 46 subjects. Gen Hosp Psychiatry. 1994;16(3):205-12.

4. Bullock K, Koran L. Psychopharmacology of compulsive buying. Drugs Today (Barc). 2003;39(9):695-700.

5. Black DW. Compulsive buying disorder: definition, assessment, epidemiology and clinical management. CNS Drugs. 2001;15(1):17-27.

6. Black DW, Monahan P, Gabel J. Fluvoxamine in the treatment of compulsive buying. J Clin Psychiatry. 1997;58(4):159-63.

7. Black DW, Gabel J, Hansen J, Schlosser S. A double-blind comparison of fluvoxamine versus placebo in the treatment of compulsive buying disorder. Ann Clin Psychiatry. 2000;12(4):205-11.

8. Ninan PT, McElroy SL, Kane CP, Knight BT, Casuto LS, Rose SE, et al. Placebo-controlled study of fluvoxamine in the treatment of patients with compulsive buying. J Clin Psychopharmacol. 2000;20(3):362-6.

9. Koran LM, Bullock KD, Hartston HJ, Elliott MA, D'Andrea V. Citalopram treatment of compulsive shopping: an open-label study. J Clin Psychiatry. $2002 ; 63(8): 704-8$

10. Aboujaoude E, Gamel N, Koran LM. A 1-year naturalistic follow-up of patients with compulsive shopping disorder. J Clin Psychiatry. 2003;64(8):94650 .

11. Koran LM, Chuong HW, Bullock KD, Smith SC. Citalopram for compulsive shopping disorder: an open-label study followed by double-blind discontinuation. J Clin Psychiatry. 2003;64(7):793-8.

12. Hollander E, Allen A. Is compulsive buying a real disorder, and is it really compulsive? Am J Psychiatry. 2006;163(10):1670-2

13. Comings DE, Blum K. Reward deficiency syndrome:genetic aspects of behavioral disorders. Prog Brain Res. 2000;126:325-41.

\section{论 Correspondência}

Hélio Tonelli

Av. Candido de Abreu, 526/311, Torre B, Centro Cívico

CEP 80530-090, Curitiba, PR

E-mail: hatonelli@terra.com.br 
Aceito em 07/08/2007.

* Este estudo foi realizado no Hospital Nossa Senhora da Luz da Aliança Saúde, Pontifícia Universidade Católica do Paraná, (PUCPR), Curitiba, PR. 